\title{
Immune Thrombocytopenia in Adult Celiac Disease
}

\author{
Hugh James Freeman* \\ Department of Medicine (Gastroenterology), University of British Columbia, Vancouver, BC, Canada \\ *Corresponding author: hugfree@shaw.ca
}

\begin{abstract}
Celiac disease has been associated with a number of immune-mediated hematologic disorders, including idiopathic or immune thrombocytopenic purpura (ITP). Review of the literature suggests that children are particularly affected, often with overt skin findings, but in this report, a 39-yr old male with biopsy-defined celiac disease was detected with a persistent immune-mediated reduction in his platelet count despite treatment and follow-up for more than a decade. Although the celiac disease resolved with a strict gluten-free diet, there was no effect on the ITP. Experience suggests that ITP may be rarely appreciated in adults (compared to children) because it may be limited in severity and occurs without clinically obvious purpura or evidence of bleeding, even with diagnostic or therapeutic biopsy procedures. A subsequent retrospective review of over 300 celiac disease patient records from a single teaching hospital clinical practice experience spanning almost 40 years confirmed no additional cases of thrombocytopenia. ITP appears to be rarely associated with celiac disease in adults and, in spite of resolution of the enteropathy, did not appear to respond to a gluten-free diet, even after more than a decade of dietary treatment.
\end{abstract}

Keywords: platelets, immune thrombocytopenia, idiopathic or immune thrombocytopenic purpura (ITP), adult celiac disease

Cite This Article: Hugh James Freeman, "Immune Thrombocytopenia in Adult Celiac Disease." International Journal of Celiac Disease, vol. 6, no. 1 (2018): 30-32. doi: 10.12691/ijcd-6-1-7.

\section{Introduction}

Celiac disease is an immune mediated small intestinal mucosal disorder, often associated with diarrhea and weight loss [1]. The disorder responds to treatment with a strict gluten-free diet. In some, malabsorption causing nutrient deficiencies may result. In addition, different immune-mediated disorders may be associated with celiac disease. Among these, hematologic disorders may develop, including an idiopathic or immune-mediated thrombocytopenia (ITP), sometimes with overtly visible purpuric skin lesions [2,3,4]. In one report [5], long-term therapy with a gluten-free diet was reported to be associated with resolution of thrombocytopenia further suggesting a significant immune-mediated linkage.

Indeed, celiac disease may result in thrombocytopenia through other mechanisms, not necessarily directly related to an immune-mediated pathogenesis. For example, some nutrient deficiencies (eg., impaired folic acid absorption) may result in impaired bone marrow generation of platelets (as well as red and white blood cells). In addition, copper deficiency related to reduced copper absorption may lead to thrombocytopenia [5]. Finally, other concomitant or coincidental causes may be present, including immunoglobulin A deficiency and either a drug-induced or a viral infection associated with thrombocytopenia $[1,6]$.

The present report documents persistent immune thrombocytopenia in an adult with celiac disease. Although the enteropathy responded completely to a gluten-free diet alone, the thrombocytopenia persisted. A subsequent retrospective evaluation of over 300 additional adults with celiac disease failed to detect thrombocytopenia. In the present patient here, diagnostic and therapeutic biopsy studies were not prohibited.

\section{Case Report}

A 39-yr-old male competitive wrestler was evaluated in another hospital for diarrhea accompanied by weight loss of $10 \mathrm{~kg}$ in 1996 . He was not using medications or dietary supplements. Blood studies (hemoglobin, white blood count) were normal except for a reduced platelet count of 117 giga per L (normal, 125 to 350 ). Other studies including an INR, calcium, phosphate, liver chemistry tests, iron, red cell folate, vitamin B12, copper and TSH were normal. Quantitation of immungloblulins ( $\mathrm{IgG}, \mathrm{IgG}$, IgM) were normal. Fecal studies for a bacterial or parasitic agent were negative. Colonoscopy and biopsies were normal. UGI endoscopy and biopsies of small intestine showed inflammatory change in the duodenal mucosa with severe mucosal "flattening" and hyperplastic crypt villus atrophy, increased mitotic figures and increased numbers of intra-epithelial lymphocytes (i.e., Marsh 3), typical of untreated celiac disease. He was treated with a gluten-free diet alone. During the next year, his symptoms improved dramatically with resolution of his diarrhea and weight gain.

In 2001, he was referred again for review. He felt completely well and blood studies were done. His hemoglobin was $160 \mathrm{G}$ per L (normal 135 to 180 ) with a normal white blood count, however, his platelet count 
remained persistently low, 122 giga per L (normal, 150 to 400). Iron studies, folic acid and vitamin B12 levels were normal. Immunglobulin quantitation as well as IgA tissue transglutaminase antibodies were normal ( 8 units). Repeat UGI endoscopic evaluation was normal and duodenal biopsies were normal. He denied any unusual bleeding or skin lesions, but platelet antibodies were positive. He was re-evaluated in 2008. All studies were normal except for a low platelet count of 134 giga per L (normal, 150 to 400 ). He remained on a gluten-free diet. In 2010, he noted some blood in his stool. Blood studies were normal except for a platelet count of 115 giga per L. Colonoscopy revealed a small polyp that was easily resected without bleeding (pathology, tubular adenoma with focally high grade dysplasia). Hemorrhoids were also present. In 2011, blood studies were normal except for his platelet count, now 124 giga per L. An IgA tissue transglutaminase antibody assay was normal while repeat colonoscopy, UGI endoscopic studies along with duodenal and colonic biopsies were normal.

\section{Added Retrospective Studies}

Subsequent to detection of this case, records for over 300 cases of adult celiac disease were retrospectively reviewed for evidence of temporary or persistent thrombocytopenia. None were found.

\section{Discussion}

Celiac disease and long-standing persistent thrombocytopenia were documented in this patient over more than a decade. In contrast to a previous report [7], thrombocytopenia was chronic and persisted despite successful management of celiac disease with a gluten-free diet. The gluten-free diet appeared to have no effect on the platelet count despite more than a decade of gluten-free diet treatment. In celiac disease, specific factors associated with persistently reduced platelet counts include immunoglobulin A deficiency (not present here), and nutritional changes related to chronic nutrient malabsorption including reduced uptake of folic acid, vitamin B12 or copper. In spite of multiple determinations, all studies were measurably normal despite ongoing, but fortunately, limited abnormal reductions in the platelet count over about 15 years. In celiac disease, superimposed, but transient causes of thrombocytopenia should also be considered, including drug-induced thrombocytopenia or a superimposed viral infection, not present in this case.

A number of case reports have documented immune-mediated thrombocytopenia with celiac disease, most often, it appears, in children, rather than in adults $[2,3,4]$. Sometimes, an autoimmune hemolytic anemia was also present (i.e., Evan's syndrome), or other autoimmune disorders, including autoimmune thyroiditis [6]. In one series of 22 children with celiac disease [8], thrombocytopenia was often the initial hematological abnormality that resulted in a later diagnosis of celiac disease, including a patient presenting with thrombocytopenia alone. In another series [9], 31 children with immune thrombocytopenia were reported to have histologically confirmed celiac disease. Interestingly, in some of these, detection of celiac disease occurred after a prolonged period, often years, and frequently, as an extra-intestinal manifestation of celiac disease without intestinal symptoms, including diarrhea. In the present adult case, it is possible given the limited degree of reduction in the platelet counts over many years that detection of immune thrombocytopenia was delayed until adulthood.

Some studies have attempted to further define the frequency of ITP in celiac disease. In our cohort derived from a single clinical practice over many decades, only a single case was found. For all of these, hematological parameters, including platelet counts, were obtained prior to intestinal biopsy studies. A further study from another centre evaluating children suggested that the frequency of immune thrombocytopenia in celiac disease may be no different from a similar paediatric control population [10]. Interestingly, in this latter report, multiple types of immune thrombocytopenia were described in celiac disease, including acute or chronic forms of ITP. These series contrast with an evaluation using national data bases in Sweden [11]. Although based on coded diagnoses, a positive association between celiac disease and immune thrombocytopenia, particularly of the chronic type, was reported, specifically for inpatients with a diagnosis of celiac disease. It is not clear if these data could be extended to all patients with celiac disease since the vast majority of celiac disease patients do not require hospitalization for investigation or treatment. As such, further studies are needed to determine if an immune-mediated thrombocytopenia occurs with an increased frequency in adults with celiac disease and whether the detection of thrombocytopenia in this age group is clinically significant or prohibits diagnostic or therapeutic biopsy.

\section{References}

[1] Freeman HJ. Celiac Disease 2017. Reference Module in Biomedical Sciences. Elsevier. 6- Dec-17.

[2] Stene-Larsen G, Mosvold J, Ly B. Selective vitamin B12 malabsorption in adult celiac disease. Report on three cases with associated autoimmune diseases. Scand J Gastroenterol 1988; 23: 1105-1108.

[3] Stenhammar L, Ljunggren CG. Thrombocytopenic purpura and celiac disease. Acta Pediatr Scand 1988; 77: 764-766.

[4] Sarbay H, Sarbay BC, Akin M, Kocamaz H, Tosun MS. Celiac disease presenting with immune thrombocytopenia purpura. Case Rep Hematol 2017; Article ID 6341321.

[5] Halfdanarson TR, Kumar N, Hogan WJ, Murray JA. Copper deficiency in celiac disease. J Clin Gastroenterol 2009; 43: 162-164.

[6] Dogan M, Sal E, Akbayram S, Peker E, Cesur Y, Oner AF Concurrent celiac disease, idiopathic thrombocytopenia purpura and autoimmune thyroiditis: a case report. Clin App Thromb Hemost 2011; 17: E13-E16.

[7] Hammami S, Haddad S, Lajmi K, Besbes LG, Meriem CB Chouchane S, Guediche MN. Immune thrombocytopenia purpura and celiac disease. J Pediatr Child Health 2011; 47: 240.

[8] Fisgin T, Yerali N, Duru F, Usta B, Kara A. Hematologic manifestation of childhood celiac disease. Acta Hematol 2014; 111: 211-214.

[9] Bansal D, Trehan A, Gupta MK, Varma N, Marwaha RK. Serodiagnosis of celiac disease in children referred for evaluation of anemia: a paediatric hematology unit's experience. Indian J Pathol Microbiol 2011; 54: 756-760. 
[10] Sarbay H, Kocamaz H, Akin M, Ozhan B. Investigation of celiac disease followed by immune thrombocytopenia purpura diagnosis in patients and comparison with literature. North Clin Istanb 2017; 4: $160-164$.
[11] Olen O, Montgomery SM, Elinder G, Ekbom A, Ludvigsson JF. Increased risk of immune thrombocytopenia purport among inpatients with celiac disease. Scand J Gastroenterol 2008; 43: 416-422. 